\section{CIENCIA E INNOVACIÓN: UNA RELACIÓN COMPLEJA Y EVOLUTIVA}

\author{
Ignacio Fernández de Lucio \\ Jaider Vega Jurado \\ Antonio Gutiérrez Gracia \\ INGENIO (CSIC-UPV), Universidad Politécnica de Valencia, \\ Camino de Vera s/n, 46022 Valencia, España
}

\section{SCIENCE AND INNOVATION: A COMPLEX AND EVOLUTIVE RELATION}

\begin{abstract}
The increasing importance of knowledge in contemporary societies is necessary to understand and analyze the relation between science and innovation today. This article is focused on how our conception of the processes of knowledge production and the process of innovation has evolved. The relation between science and innovation has changed compared to past decades, nowadays the concept of innovation and its' relation with science goes beyond the application of the scientific method inside the scientific community. This relation extends itself into other spheres such as the cultural and the social where other applications are involved and where the development of public policy becomes crucial.
\end{abstract}

KEY WORDS: Knowledge; innovation; science; public policy; society.

\section{INTRODUCCIÓN}

Existe un consenso generalizado con relación a la importancia que tiene el conocimiento como factor de desarrollo en las sociedades contemporáneas. Desde hace ya varias décadas se ha venido señalando que el crecimiento económico de una nación depende cada vez menos de la acumulación de los factores productivos tradicionales (tierra, capital y trabajo) y se asocia, cada vez más, a la capacidad que ésta tenga para generar y aplicar nuevos conocimientos, derivando en lo que algunos autores han descrito como la emergencia de las economías basadas en el conocimiento. Esta expresión, ampliamente utilizada para dar cuenta de lo que se considera el rasgo fundamental de la sociedad actual, puede, no obstante, llevar erróneamente a pensar en el progreso científico como condición
RESUMEN: La cada vez mayor importancia que está adquiriendo el conocimiento en las sociedades contemporáneas hace necesario profundizar en el análisis de la relación existente entre ciencia e innovación. Este artículo la aborda considerando como ha evolucionado nuestra concepción sobre los procesos de producción de conocimiento y el proceso innovador. La revisión bibliográfica realizada muestra que la emergencia de nuevos enfoques en estos campos ha generado una visión sobre la relación ciencia-innovación bastante diferente a la que predominaba hace algunas décadas. De esta forma, si bien por un lado se reconoce que el proceso innovador no depende exclusivamente del desarrollo de actividades científicas, por otro, se establece que el vínculo ciencia-innovación puede ser hoy mucho más estrecho, teniendo en cuenta que las actividades de investigación se desarrollan, cada vez más, en un contexto de aplicación. En el artículo se discute el alcance real de esta nueva visión y las implicaciones que tiene para el diseño de políticas públicas.

PALABRAS CLAVE: Conocimiento; innovación; ciencia; políticas públicas; sociedad.

necesaria y suficiente para el desarrollo económico de un territorio, olvidando el papel que desempeña la innovación y los procesos de aprendizaje social asociados a la misma. En este sentido, si algo ha demostrado la dinámica del desarrollo de los territorios en los últimos años es que el éxito de una economía depende no sólo de su excelencia científica, sino también de su capacidad para introducir nuevas combinaciones en las actividades productivas. Por ello, quizá resulte más conveniente, tal como lo aconsejan Arocena y Shutz (2001), hablar de una "economía basada en el conocimiento, modelada por el aprendizaje y motorizada por la innovación".

El conocimiento y la innovación aparecen, por lo tanto, como dos elementos consustanciales al desarrollo de las sociedades modernas, por lo que su estudio y análisis ha 
adquirido relevancia en los últimos años. De hecho, como fruto del renovado interés en estos aspectos, en las últimas décadas se han desarrollado nuevos enfoques que tratan de explicar la forma en que se organizan y ejecutan las actividades científicas y la naturaleza misma del proceso innovador. Así, por ejemplo, la visión sobre el funcionamiento y organización de la ciencia ha exhibido un giro importante, reconociendo, entre otras cosas, una mayor dependencia de las actividades científicas hacia factores externos de carácter social, económico o político. Como resultado de ello, el modelo de ciencia tradicional, caracterizado por un alto grado de autonomía y guiado por intereses académicos y disciplinarios, ha dado paso a un modelo de ciencia mucho más regulado, en el cual tanto la agenda de investigación como los sistemas de incentivos y evaluación son definidos atendiendo cada vez más a las demandas y necesidades externas, no necesariamente convergentes con los intereses o valores académicos.

De forma similar, la visión sobre la naturaleza del proceso innovador ha experimentado una transformación sustancial. Hasta los años setenta del siglo pasado se admitía que la innovación descansaba fundamentalmente en la aplicación de los conocimientos científicos y que el proceso de traducción de dichos conocimientos se desarrollaba de una forma lineal y secuencial a través de fases discretas, tanto en el plano temporal como institucional. Sin embargo, la no correspondencia entre los gastos en I+D y los resultados económicos de los países de la OCDE en dicha década llevaron a diferentes autores a considerar que los resultados en materia de innovación de una economía no son una consecuencia directa de sus resultados científicos y a destacar la importancia que tienen otras fuentes de conocimiento en el proceso innovador así como su carácter interactivo.

Estos nuevos enfoques sobre los procesos de producción y aplicación de conocimiento son utilizados actualmente como base para explicar el papel de la ciencia, no sólo en la innovación, sino también en el desarrollo económico y social de los territorios. De esta forma, si bien por una parte se reconoce que el proceso innovador no depende exclusivamente del desarrollo de actividades científicas, por la otra, se establece que el vínculo ciencia-innovación puede ser hoy mucho más estrecho, teniendo en cuenta que las actividades de investigación se desarrollan, cada vez más, en un contexto de aplicación. Estos enfoques han recibido importantes adhesiones en el ámbito político, pero han generado al mismo tiempo un intenso debate en el ámbito académico sobre la validez y alcance de los mismos. De hecho, ¿hasta qué punto estos enfoques dan cuenta de la forma en que se llevan a cabo los procesos de producción de conocimiento e innovación en el conjunto de los diferentes sistemas científicos e industriales? y, quizá más importante aún ¿Qué repercusión tiene dichos enfoques en la relación entre ciencia e innovación? Son algunas de las cuestiones que permanecen abiertas.

Como corolario a lo anteriormente mencionado, es posible afirmar que la relación entre ciencia e innovación no sólo adquiere hoy mayor importancia como elemento de análisis, sino que además reviste un mayor grado de complejidad. Este artículo pretende abordar esta relación aproximándose desde dos perspectivas diferentes, pero estrechamente interrelacionadas. La primera perspectiva explora el papel que desempeña la aplicación del conocimiento en los procesos de generación de dicho conocimiento. La segunda perspectiva focaliza el análisis en el papel que juega la ciencia en los procesos de innovación tecnológica, es decir, el papel de la ciencia como fuente de innovación. De la primera perspectiva dan cuenta fundamentalmente los enfoques sociológicos sobre la ciencia, mientras que la segunda está más relacionada con los enfoques sobre el proceso innovador desarrollados en el campo de la economía y la gestión de empresas. En ambos casos, tal como se ha mencionado, se ha evidenciado una evolución en las visiones dominantes, lo cual si bien ha servido para responder algunos interrogantes, ha generado nuevas dudas con respecto a la naturaleza de la relación ciencia-innovación. En este sentido, la estrategia de análisis empleada en este artículo parte de la premisa que para entender la relación ciencia-innovación es necesario conocer cómo han evolucionado las concepciones sobre las mismas a lo largo de estos últimos años y discernir el valor que tienen los enfoques actuales cómo marcos explicativos así como su aplicabilidad práctica real.

\section{LOS ENFOQUES SOBRE LOS PROCESOS DE PRODUCCIÓN DE CONOCIMIENTO}

Desde el campo de la sociología se han desarrollado diversos enfoques que tratan de caracterizar los procesos 
de producción y validación del conocimiento, así como los rasgos que definen la ciencia como institución socialmente organizada. Estos estudios, que han dado cuerpo a la denominada sociología de la ciencia, pueden servir de base para realizar una primera aproximación al análisis de la relación existente entre ciencia e innovación, explorando cómo la utilidad o aplicabilidad del conocimiento influye en la organización de las actividades científicas.

Hablar sobre los estudios sociales de la ciencia conlleva necesariamente a hacer referencia a los trabajos de Robert K. Merton, quien durante la primera mitad del siglo pasado identificó los elementos que constituyen el ethos de la ciencia y que han permitido su desarrollo y mantenimiento a lo largo del tiempo. Estos elementos, que a juicio de Merton tienen un carácter imperativo y obligatorio, son característicos de la ciencia pura y se concretan en los valores de comunismo, universalismo, desinterés y escepticismo organizado (Merton, 1942). La primera letra de cada una de estas palabras produce el acrónimo en inglés CUDOS, que fonéticamente suena como el término griego kudos, que significa fama derivada de un logro o descubrimiento y que sintetiza magistralmente lo que persigue el investigador en esta concepción de la ciencia.

Los valores de esta ciencia pura pueden caracterizarse de la manera siguiente. El universalismo destaca que los criterios de la ciencia y no los atributos personales del científico deben ser las bases para juzgar los méritos de la labor científica. En este sentido, la aceptación o rechazo de los hallazgos de la ciencia no deben depender de los atributos personales o sociales de los investigadores, sino de los argumentos o pruebas que se presenten. El comunismo exige la difusión accesible y pública de los resultados no sólo a la comunidad científica, sino también a la sociedad como un todo. Este valor parte de la idea que los resultados de investigación son producto de la colaboración social y, por lo tanto, tienen que ser asignados a la comunidad. El desinterés, por su parte, dirige al científico a centrarse en el avance de la ciencia y no en los intereses y prejuicios personales. Atendiendo a este principio, el científico no debe aspirar a más beneficio que aquél que proporciona el hecho de contribuir al desarrollo de su comunidad y la satisfacción del trabajo realizado. Por último, el escepticismo organizado, fomenta el debate y la evaluación crítica de unos científicos a otros y sugiere que la aceptación o rechazo de los resultados e ideas de investigación se basen en la evaluación por pares y no en la tradición o en la autoridad vigente.

Para Merton, los valores anteriores, aunque no se encontraban explícitamente definidos, podían inferirse de la conducta de los investigadores y se reforzaban tanto por la socialización que sufren quienes entran a formar parte de la comunidad académica, como por las sanciones que se aplicaban a quienes los trasgueden (Fernández y Torres, 2009). Merton complementa esta descripción del ethos científico con el desarrollo de su tesis sobre el intercambio social, según la cual la ciencia se estructura y se refuerza a partir de un sistema que otorga al científico una recompensa en función del aporte que éste realiza al fondo común de conocimiento. Dicha recompensa se materializa no sólo en términos de reconocimiento por parte de la comunidad científica, sino también a través de la asignación de recursos económicos.

El pensamiento mertoniano sobre las normas y valores de la ciencia estableció las bases de lo que podría denominarse el paradigma clásico de la sociología de la ciencia. Atendiendo a esta visión, el desarrollo de la ciencia estaba regulado básicamente por intereses académicos y disciplinarios, siendo la originalidad y la excelencia científica los criterios básicos utilizados para producir y valorar el conocimiento. Las consideraciones de tipo utilitarista, relacionadas con la aplicación o explotación socio-económica del conocimiento, no tenían mayor peso en la estructuración social de la ciencia, ni en el desempeño de los colectivos que la hacían funcionar. No se quiere decir con ello que en el marco de esta visión no se reconociese valor práctico a la ciencia, pero sí que la preocupación sobre este aspecto era un hecho que trascendía la dinámica interna de la comunidad cientifica. En este sentido, y con respecto al tema que nos ocupa, es posible decir que de esta visión se desprende una relación indirecta entre ciencia e innovación. Si bien el conocimiento científico podría ser aplicado para la resolución de algunos problemas prácticos, este tipo de consideraciones no influía ni en la organización de la ciencia ni en el proceso mismo de producción del conocimiento. El objetivo central de la ciencia era el incremento progresivo del conocimiento, el cual, a su vez, constituía un bien común. La comunidad científica, por lo tanto, funcionaba como un sistema autónomo, regulado básicamente por lógicas internas y que no exhibía una relación directa con el entorno social y sus expectativas. 
Las tesis de Merton tuvieron notables implicaciones no sólo en la organización y evaluación de la actividad científica sino también en el diseño de políticas públicas durante la segunda mitad del siglo XX. No obstante, a partir de la década de 1970 surgen diversos trabajos que critican los pilares del ethos científico mertoniano y dan lugar a lo que algunos autores denominan un nuevo institucionalismo en la sociología de la ciencia (Mitroff, 1974; Mulkay, 1976; Sklair, 1972). Estos trabajos se caracterizan no sólo por poner en tela de juicio la validez de las normas y valores identificados por Merton, sino también por prestar mayor atención a los comportamientos de las organizaciones que producen ciencia, considerando tanto su faceta interna como su relación con otros actores. Focalizando la atención en este último aspecto, dos de las interpretaciones más conocidas son probablemente la noción de la ciencia posacadémcia y la tesis del modo 2".

La noción de "ciencia posacadémica" ha sido desarrollada por John Ziman para caracterizar lo que a juicio del autor corresponde a una "transformación radical e irreversible de la forma en la que la ciencia se organiza, se gestiona y se realiza alrededor del mundo" (Ziman 2000, 7). Según este autor, en las últimas décadas del siglo pasado la ciencia académica, idealizada en los principios clásicos mertonianos, sufrió una transformación sustancial que la acercó mucho más a las prácticas de la ciencia industrial. Esta transformación tuvo como causa fundamental el incremento en la competición por fondos destinados a la investigación, lo que generó un control más estricto de la gestión pública de la actividad científica e incrementó la rendición de cuentas por parte de los científicos ante la sociedad. Como consecuencia de ello, los CUDOS son alterados por cinco normas mucho más propias de la investigación tecnológica realizada en las empresas, que se resumen en el acrónimo PLACE, que significa en inglés posición o puesto, y cuya elección es ingeniosa para comparar al CUDOS de la ciencia pura. Las nuevas normas son: Propietaria (en lugar de comunal), local (en lugar de universal), autoritaria (en lugar de desinteresada), comisionada (en lugar de original) y de expertos (en lugar de escépticos). En este sentido, se entiende que los resultados de investigación están pasando a ser de propiedad y, por tanto, no son necesariamente públicos o comunitarios. En segundo lugar, los investigadores se centran en problemas locales que no contribuyen a la comprensión y el entendimiento general. En tercer lugar, los investigadores actúan bajo una autoridad de gestión, más que como investigador individual. En cuarto lugar se observa que el trabajo científico es comisionado a los investigadores para resolver determinados problemas y no para contribuir al conocimiento como un todo. Finalmente, el científico se valora por su capacidad como experto que soluciona problemas y no por su creatividad personal'.

Para Ziman, uno de los rasgos fundamentales que se deriva de la emergencia de la ciencia posacadémica es que la actividad científica se convierte en un proyecto social más colectivo, en la medida en que admite la participación de grupos u organizaciones ajenas al quehacer científico en la definición de los problemas de investigación. Ello, obviamente, penaliza la autonomía y el individualismo que caracteriza a los científicos académicos tradicionales y hace la actividad cientifica más permeable a las demandas y exigencias del entorno. Desde este punto de vista, la agenda de investigación se configura obedeciendo no sólo a intereses académicos sino también teniendo en cuenta intereses sociales mucho más amplios. La utilidad de los resultados de investigación llega a ser, por lo tanto, un aspecto central en los procesos de producción de conocimiento.

En paralelo a la noción de ciencia posacadémica, aparece la tesis del Modo 2 de producción del conocimiento, recogida en dos obras clave desarrolladas por Gibbons et al. (1994) y Nowotny et al. (2001). Según esta tesis, en la sociedad contemporánea, junto a la modalidad habitual de la ciencia académica, disciplinaria, organizada en forma jerárquica, homogénea y con estructuras básicamente estables (Modo 1) tiene lugar otra forma de producción de conocimientos, que es transdisciplinar, heterogénea, poco jerárquica y estructuralmente cambiante (Modo 2). Así, mientras que en el Modo 1 la producción de conocimiento obedece a las normas cognitivas que rigen cada disciplina y se desarrolla con el objetivo de avanzar en el conocimiento de la realidad para satisfacer los propios intereses académicos, en el Modo 2 el conocimiento se genera siempre en el contexto de la aplicación, es decir, atendiendo a las necesidades explícitas de algún agente externo. En este punto es necesario advertir, que el término aplicación no se refiere simplemente al hecho de que el conocimiento se produce como respuesta a una demanda específica, ya sea de la industria, de la sociedad o del mercado, sino que es el resultado de un proceso en 
el cual confluyen numerosos factores que representan mucho más que simples consideraciones comerciales. En el Modo 2, la ciencia ha ido más allá del mercado y la producción de conocimientos llega a ser difundida a través de la sociedad, convirtiéndose en un proceso socialmente distribuido (Gibbons et al., 1994).

La tesis del Modo 2 destaca también que la validación de los resultados de investigación descansa fundamentalmente en su aceptación social y en su relevancia para la satisfacción de necesidades específicas, más que en la contribución al avance del fondo común de conocimiento. Se habla así de un conocimiento robusto socialmente que es valorado no sólo por una comunidad científica, sino también por diseminadores, comerciantes y usuarios finales del mismo (Nowotny et al., 2001).

A pesar de las diferencias de aproximación que existen entre la noción de ciencia posacadémica y la tesis del Modo 2, estos enfoques transmiten la misma idea general: la emergencia de una nueva manera de hacer ciencia que, en comparación con la de la ciencia académica tradicional, ve modificada no sólo su sociología interna sino también su función social externa. Tanto el concepto de colectivización de la ciencia, como el de producción de conocimiento en el contexto de aplicación vienen a destacar la utilidad del conocimiento como otra dimensión fundamental que debe ser tenida en cuenta en los objetivos y desarrollo de las actividades científicas. De estos enfoques se desprende, por lo tanto, la imagen de una relación mucho más estrecha entre ciencia e innovación, en la medida en que el conocimiento se produce considerando no sólo su valor intrínseco sino la utilidad del mismo y la investigación se orienta cada vez más hacia valores sociales, económicos e incluso políticos. Se desarrolla así una especie de vínculo inverso -inexistente en el esquema de ciencia académica tradicional- a través de la cual la sociedad interviene en el tipo de conocimiento que se produce y lo legitima en función de los beneficios sociales y económicos esperados.

En el marco de estos nuevos enfoques, la idea de una relación estrecha entre ciencia e innovación se ve también reforzada por la ampliación de los agentes productores de conocimiento. La colectivización de la ciencia y el predominio de los contextos dinámicos de aplicación hacen que se multipliquen los entornos potencialmente generadores de conocimiento y acaban, en gran parte, con el monopolio que ostentaban las organizaciones científicas tradicionales (universidades, organismos públicos de investigación) en este proceso. Esta ampliación se manifiesta no sólo en la participación directa de empresas y consultoras en la generación de conocimiento, sino también en el desarrollo de organizaciones híbridas que trascienden las fronteras institucionales y se orientan a la explotación socio-económica del conocimiento generado. Se desarrolla de esta forma una gran heterogeneidad en cuanto a la forma en que se organizan las actividades científicas, la forma como se llevan a cabo los trabajos de investigación y los actores que participan en el proceso.

\section{El papel de la ciencia en la innovación}

Otra forma de analizar la relación ciencia-innovación es considerando como ha evolucionado el papel que se atribuye a las actividades científicas dentro del proceso innovador. Ello conlleva a analizar la evolución de los diferentes enfoques existentes en la literatura con relación a la naturaleza, fuentes y determinantes de la innovación.

Joseph Schumpeter fue el primer autor en ofrecer una amplia definición de innovación y en destacar su importancia en la dinámica del crecimiento económico. Este autor definió la innovación como la introducción de "nuevas combinaciones" de los recursos productivos, las cuales podían adoptar cinco formas básicas: a) la introducción de un nuevo producto; b) la introducción de un nuevo proceso; c) la apertura de un nuevo mercado; d) la conquista de nuevas fuentes de suministro de materias primas, y e) los cambios en la organización industrial. En su libro Business Cycles (1939), Schumpeter resaltaba el impacto potencial que las innovaciones ejercen sobre el conjunto de la economía y adicionalmente postulaba la existencia de dos tipos de individuos: los empresarios innovadores que, incapaces de prever el futuro, están dispuestos a enfrentarse a todos los riesgos para llevar a cabo innovaciones y los imitadores que simplemente actúan como gestores rutinarios siguiendo el camino abierto por los heroicos pioneros (Freeman, 1998). Asimismo, Schumpeter concebía la innovación como un proceso de destrucción creadora a través del cual se reconfiguraba constantemente la estructura de la industria. 
Si bien Schumpeter no desarrolló un modelo formal sobre cómo se lleva a cabo el proceso innovador, ni tampoco dedicó mucha atención a identificar el rol que desempeña la ciencia en el mismo (Maclaurin, 1953), planteó una distinción que serviria de base para avanzar en este campo. Schumpeter subrayó la necesidad de distinguir entre invención e innovación. La primera está representada por la producción de un nuevo conocimiento y es generalmente el resultado de las actividades científicas, mientras que la segunda está asociada con la primera aplicación o explotación comercial del conocimiento. La invención, por lo tanto, carece de valor económico en sí misma y no es una condición suficiente para la innovación. Además de estas dos categorías Schumpeter distinguió también la innovación de la difusión, siendo esta última el proceso a través del cual la innovación es adoptada por otros agentes económicos distintos a los que la han desarrollado. Aunque Schumpeter profesó poca dependencia de la innovación con respecto a la invención ${ }^{2}$ la secuencia invención-innovación-difusión fue empleada posteriormente por otros autores para describir el proceso innovador. Es así como fueron surgiendo, a partir de la década de 1950, diferentes modelos teóricos que, a pesar de sus diferencias, se caracterizaban por presentar la innovación como una secuencia de actividades o fases que tenía como punto de partida la generación de nuevo conocimiento, continuaba con la aplicación y explotación comercial de dicho conocimiento y culminaba con la difusión. Esta interpretación de la innovación es lo que en términos genéricos se ha denominado en la literatura como el "modelo lineal de innovación".

El origen de este modelo es un tema que se ha debatido ampliamente en la literatura sin que aún exista un consenso general sobre ello. Una de las versiones más extendidas a este respecto es la que reconoce las bases de este planteamiento en el informe que Vannevar Bush presentó al presidente Roosevelt, titulado Ciencia, la frontera sin fin, (Bush, 1945). En dicho informe el autor destaca el papel central que el conocimiento científico desempeña en el progreso industrial y la necesidad de generar una sólida base científica nacional como requisito para el desarrollo económico del país. Este autor expresó que si se desea lograr algo tan concreto como generar nuevos puestos de trabajo en un pais o desarrollar nuevos productos y procesos, es preciso invertir en investigación básica, ya que ésta, a través de diversas mediaciones, garantiza el logro de tal objetivo. En otras palabras, identificó como origen de las innovaciones el conocimiento generado a través de la investigación básica. Dicha investigación, asimismo, debía ser apoyada y desarrollada asegurando la libertad del científico para definir sus áreas de trabajo y guiada por el objetivo de aumentar el acervo de conocimiento existente. De hecho, Bush destacó la necesidad de que los científicos trabajen en temas de su propia elección, según la manera que les dicte su curiosidad por la exploración de lo desconocido y libre de lo que él llamó la "presión adversa de la convención, el prejuicio o la necesidad comercial" (Bush, 1945). En este sentido, el autor estableció que la aplicación y explotación económica del conocimiento generado a través de la investigación básica se llevaría a cabo en otros ámbitos y a través de otras actividades, como por ejemplo la investigación aplicada desarrollada en los laboratorios empresariales de I+D.

Teniendo en cuenta lo expresado precedentemente, puede decirse que el informe de Bush reforzaba el carácter inmaculado de la actividad científica, la cual debía orientarse hacia la generación de conocimiento sin preocupación aparente por el carácter instrumental del mismo. La aplicación de dicho conocimiento, si bien se reconocía como un elemento importante, constituia una preocupación que se escapaba de los objetivos de los científicos y se tenía que gestionar en otras esferas. En este sentido, aunque el planteamiento de Bush tiene claramente un carácter más económico que sociológico, presenta muchas semejanzas con los principios de organización y ejecución de la ciencia identificados por Merton. Ello puede obedecer a la existencia de una mutua influencia entre estos autores o simplemente a que esta visión de la ciencia formaba parte del acervo cultural de aquella época.

Aunque en el informe de Bush hay una descripción de la importancia de la ciencia para el progreso socio-económico, algunos autores consideran que en dicho informe no se encuentra una construcción explícita del modelo lineal de innovación, por lo menos en toda su extensión. Godin (2006), por ejemplo, sostiene que lo más lejos a lo que llegó Bush fue a establecer una relación entre investigación básica e investigación aplicada, pero en ningún momento planteó o desarrolló un argumento basado en un proceso secuencial a través del cual la ciencia genera beneficios económicos. En este sentido, este autor sostiene que el modelo lineal de innovación difícilmente puede ser catalogado como la invención espontánea surgida de la 
mente de un solo individuo, sino que representa más bien el producto de una línea de pensamiento en la que convergieron especialistas de distintas comunidades científicas a lo largo de varias décadas. Primero fueron los científicos naturales que desarrollaron la retórica sobre la investigación básica como fuente de la investigación aplicada o la tecnología. Segundo, fueron los investigadores industriales que estudiaron la gestión industrial de la investigación y el desarrollo de tecnología. Tercero, los economistas que introdujeron el concepto de innovación. Estas tres comunidades fueron incorporando su propio término al modelo (investigación, desarrollo tecnológico, innovación) culminando así la secuencia (Godin, 2006).

Independientemente de su origen, el modelo lineal se convirtió en el enfoque por excelencia para explicar el proceso innovador desde finales de la década de 1940. En su versión extendida, los principios básicos que transmite este enfoque pueden describirse como sigue:

a) El proceso innovador tiene lugar a través del desarrollo de una serie de etapas sucesivas, que siguen un flujo unidireccional sin retroalimentaciones con los pasos previos. Este proceso se desarrolla de manera progresiva a través del eje: investigación básica - investigación aplicada - desarrollo tecnológico - Innovación - difusión.

b) La fuente de las innovaciones proviene, por lo tanto, de los avances en el conocimiento científico, el cual se transforma en tecnologías aplicables a nuevos productos y a la puesta en marcha de nuevos procesos.

c) Esta transformación es fluida, pero tiene lugar en ámbitos diferentes. De esta forma, existe una clara división del trabajo a lo largo de toda la secuencia, en la que participan diferentes agentes que actúan atendiendo lógicas también distintas. Así, la investigación básica es típicamente conducida en las universidades e institutos de investigación, mientras que la investigación aplicada y el desarrollo tecnológico es Ilevado a cabo en las empresas, especialmente aquéllas con un fuerte departamento de I+D.

d) Dada esta especialización del trabajo y que cada agente responde a diferentes motivaciones e incentivos, una interacción directa entre ellos (por ejemplo, entre universidad y empresa) no constituye un elemento fundamental para el desarrollo del proceso. De hecho, lo más aconsejable es garantizar la libertad y auto- nomía de cada uno de los agentes que intervienen en el mismo.

e) Este enfoque es de naturaleza tecnocrática, ya que concibe la evolución tecnológica en términos de organización de los procesos técnicos y de invenciones materiales.

De los principios anteriores se desprende una relación entre ciencia e innovación que podría ser caracterizada en términos de una relación de dependencia, pero de naturaleza indirecta. En otras palabras, la innovación depende de los avances en el conocimiento científico, pero el proceso a través del cual dicho conocimiento se transforma efectivamente en aplicaciones comerciales con valor económico no es directo, sino que por el contrario está mediado por diversas etapas y actividades. En este sentido, aunque la ciencia se reconoce como la fuente por excelencia de la innovación, la innovación poco o nada influye en la organización y ejecución de la ciencia.

Diversos trabajos realizados en el campo de la economía durante las décadas de los sesenta y setenta (Mansfield, 1968; Myers y Marquis, 1969; Utterback, 1974) reforzaron la idea que el proceso innovador podía ser adecuadamente descrito a través de la secuencia invención-innovacióndifusión ${ }^{3}$. Adicionalmente, el desarrollo de estadísticas sobre ciencia y tecnología, así como la estandarización y normalización de las definiciones y medidas empleadas en este campo -llevadas a cabo por organizaciones como la OCDE y la NSF- contribuyeron a que dicho modelo pasase de ser una manifestación retórica y se afianzase como un hecho social (Godin, 2006).

Aunque el modelo lineal llegó a ser el pensamiento convencional en materia de ciencia e innovación durante las décadas posteriores a la segunda guerra mundial, lo cierto es que no tardaron en aparecer las primeras críticas al mismo. La idea de la investigación básica como fuente principal de la innovación, si bien se afianzó en el seno de la comunidad científica como justificación para asegurar un flujo suficiente de recursos públicos, fue debatida tempranamente por investigadores y organizaciones más cercanos a la comunidad industrial. Así, por ejemplo, a finales de la década de 1960 un estudio financiado por el Departamento de Defensa de los Estados Unidos -denominado HINDSIGHT- arrojó varias dudas sobre el aporte de la ciencia para el desarrollo de innovaciones. Según este 
estudio, menos del 10\% de un conjunto de innovaciones desarrolladas en el ámbito militar se derivaban de la investigación básica, mientras que la mayoría era el resultado de proyectos tecnológicos de ingeniería (U.S. Departament of Defense, 1969) ${ }^{4}$. Las conclusiones de este estudio marcaron el inicio de una serie de críticas al enfoque lineal de la innovación que encontraron probablemente su expresión más fuerte en el artículo de Kline y Rosenberg (1986) titulado "An Overwiew of Innovation").

En el artículo anteriormente señalado, Kline y Rosenberg cuestionaron varios de los pilares del modelo lineal. En primer lugar, estos autores indicaron que la mayor parte de las innovaciones se desarrollan a través de la aplicación del conocimiento disponible y que sólo en aquellas ocasiones en las cuales el conocimiento existente resulta insuficiente para resolver un problema especifico es cuando se recurre a la investigación básica. Esta idea se deriva en gran parte del reconocimiento de la innovación como un proceso de naturaleza continua, basado más en el desarrollo de mejoras incrementales en los productos, procesos y prácticas existentes, que en la transformación radical de los mismos. Así, estos autores señalan que si bien la ciencia se encuentra generalmente en la base de las innovaciones radicales, estas últimas son solamente un pequeño porcentaje de las innovaciones que tienen lugar en una economía ${ }^{5}$. Las innovaciones incrementales son más frecuentes y se caracterizan por tener una mayor diversidad de fuentes, tanto al interior de la empresa como en su entorno ${ }^{6}$.

Otro aspecto debatido por Kline y Rosenberg fue la dirección de la relación entre ciencia e innovación. En esta línea, estos autores señalaron que muchos de los desarrollos tecnológicos de la sociedad moderna no han dependido de la investigación básica y que, además, en no pocos casos la tecnología ha proporcionado insumos importantes para la investigación científica ya sea en términos de problemas a resolver o instrumentación. En otras palabras, no sólo cuestionaron la dependencia del desarrollo tecnológico hacia la investigación, sino también plantearon que la relación entre estas actividades puede seguir un flujo inverso al descrito por el modelo lineal.

No obstante, la crítica más fuerte que Kline y Rosenberg hacen al modelo lineal se encuentra en la naturaleza misma del proceso innovador. Para estos autores, la innovación posee un carácter interactivo y complejo. No es el resulta- do de un proceso secuencial que tiene un punto de partida claramente definido, sino el producto de un conjunto de actividades que pueden tener lugar simultáneamente y, sobre todo, que se retroalimentan mutuamente. Estos argumentos se encuentran sintetizados en el conocido modelo de enlaces en cadena a través del cual los autores destacan los continuos flujos que se dan en las distintas etapas del proceso innovador (Kline y Rosenberg, 1986).

El trabajo de Kline y Rosenberg, junto con los realizados por otros investigadores en este campo, configuraron una visión sobre el proceso innovador muy diferente a la derivada del modelo lineal. En esta visión, las actividades de I+D dejan de ser consideradas el punto de partida por excelencia de la innovación y se reconoce la importancia de otras actividades (diseño, producción, marketing, etc.) como fuente de conocimiento para el desarrollo de nuevos productos y procesos. Asimismo, la innovación se contempla como un proceso interactivo, caracterizado por la existencia de continuas realimentaciones en las diferentes etapas del mismo.

Este carácter interactivo que se le atribuye al proceso innovador es probablemente el rasgo más importante de esta nueva visión de la innovación. De él se desprende importantes implicaciones, no sólo en la forma en que se organiza la innovación en el contexto de la empresa, sino también en la manera como ésta tiene lugar a nivel social. Así, mientras que en el modelo lineal se prima la autonomía e independencia de los diferentes agentes sociales, en el marco de esta nueva visión la interacción entre los mismos es un aspecto fundamental. La innovación pasa a ser considerada un proceso colectivo y dinámico, que lo lleva a cabo la empresa a través de una relación activa con otros agentes e instituciones (científicas, industriales o gubernamentales), configurando lo que se ha denominado sistema de innovación ${ }^{7}$. Atendiendo a esta visión, las universidades y en general las organizaciones científicas dejan de ser espacios aislados, activos únicamente al inicio del proceso innovador -a través de la investigación básica- y se convierten en agentes con la capacidad y necesidad de relacionarse con su entorno económico para trabajar conjuntamente en el desarrollo de innovaciones.

Focalizando la atención en el tema que nos ocupa, es posible establecer que este cambio en la visión del proceso innovador ha supuesto una transformación igualmente 
importante en la forma de entender la relación entre ciencia e innovación. Por una parte, se reconoce que la innovación puede partir de actividades diferentes a la investigación básica, pero, por otra parte, se establece que en aquellos casos en que la ciencia actúa como fuente de la innovación, las relaciones entre ellas llegan a ser, por decirlo de alguna forma, más cercanas. En estos casos, la investigación se desarrolla más cerca al campo de la aplicación y las etapas siguientes que desembocan en la innovación están cada vez más interconectadas, a diferencia de lo que postulaba el modelo lineal donde los límites entre las actividades científicas y empresariales estaban claramente identificados. Es lo que Etzkowitz y Leydesdorff (2000) han descrito como el paso de una "frontera sin fin" (parafraseando a Bush) a una "transición interminable".

\section{Discusión}

La emergencia de nuevos enfoques sobre los procesos de producción de conocimiento y el carácter interactivo del proceso innovador plantean un escenario en el cual las fronteras entre la generación y aplicación de conocimiento se tornan cada vez más difusas, lo que ha generado el desarrollo de una nueva visión en torno a la relación cienciainnovación bastante diferente a la que predominaba hace algunas décadas. Esta visión se caracteriza por la existencia de un vínculo más cercano entre ciencia e innovación, producto de la orientación de las actividades científicas hacia el contexto de aplicación y de la colectivización de los procesos de producción de conocimiento.

No obstante, convendría preguntarse por el alcance real de estos nuevos enfoques $y$, consecuentemente, la trascendencia de la relación ciencia-innovación que de ellos emerge. En este sentido, si bien los valores de la ciencia pura han sido cuestionados por considerar que constituyen una visión idealizada del mundo científico, lo cierto es que tanto la noción de la ciencia posacadémica como la tesis del Modo 2 tampoco se apoyan en análisis empíricos robustos y ambas aproximaciones son ambiguas con respecto a la sustitución de las formas tradicionales de ciencia y con relación a la posibilidad de convivencia entre los distintos modos de producción del conocimiento (Godin, 1998; Hessels y Van Lente, 2008).
La literatura que ha desarrollado los dos enfoques antes mencionados emplea un estilo ensayístico y no especifica marcos temporales, espaciales e incluso disciplinares que permitan comparar la consolidación de los nuevos patrones que ellos destacan con las formas tradicionales de hacer ciencia. A este respecto, algunos autores han señalado que la creación de conocimiento en el contexto de aplicación es una característica que siempre ha estado presente, en mayor o menor medida, en el desarrollo de la ciencia y que por lo tanto no constituye un aspecto novedoso. Los orígenes de la ciencia moderna en el siglo XVIII, por ejemplo, se encontraron al combinar la búsqueda de la solución de problemas prácticos de tipo social y económico con el deseo de adquirir conocimiento para la propia consideración (Weingart, 1997; Godin, 1998; Martin y Etzkowitz, 2000).

Además, se ha destacado la dificultad de extrapolar la tesis derivada de estos enfoques a sociedades o contextos geográficos diferentes a los empleados para su construcción. La sociedad a la que hace referencia Nowotny et al. (2001) en su obra es una sociedad concreta, donde los estados-nación están siendo socavados por abajo (por el fortalecimiento de la dimensión local y regional) y por arriba (por el desarrollo de agrupaciones supranacionales), donde no existe una clara demarcación entre las esferas público y privada y donde el avance de la ciencia y la tecnología ha expandido el territorio de lo político creando la necesidad de nuevos marcos regulatorios. Estos enfoques, por lo tanto, no han sido desarrollados pensando en el orden social de los países periféricos, por lo que su uso en estos contextos requeriría no sólo una adaptación, sino en muchos casos un rechazo a algunos de los aspectos clave que de ellos emergen (Albornoz, 2003).

Por último, pero no menos importante, se ha criticado también la capacidad que tienen dichos enfoques para describir el funcionamiento de la ciencia en su conjunto. La colectivización del proceso de producción de conocimiento, producto de una participación activa de agentes no científicos en el mismo, es un rasgo que ciertamente caracteriza algunos campos o áreas de investigación en la actualidad, pero no puede decirse que se encuentre presente en todo el sistema científico. Además, algunas de las características propias de la ciencia académica (por ejemplo, revisión por pares) tienen aún vigencia como elemento legitimador del quehacer del investigador en la 
mayoría de las comunidades científicas (Jiménez-Buedo y Ramos, 2009).

Teniendo en cuenta lo expuesto precedentemente, no parece que los valores de la ciencia posacadémica hallan sustituido mayoritariamente a los valores mertonianos de la ciencia pura. Dependiendo del ámbito disciplinar e incluso del contexto socio-económico, uno u otro tipo de valores pueden estar presentes. De hecho, en una misma institución científica (la universidad, por ejemplo), pueden coexistir tanto la lógica académica como la posacadémica.

Por otra parte, si bien el enfoque interactivo ha contribuido mucho a mejorar nuestro entendimiento sobre la naturaleza del proceso innovador y la dinámica de las organizaciones que en él participan, algunos de los postulados del modelo lineal pueden seguir teniendo validez en contextos específicos. Así, por ejemplo, aunque la ciencia no sea la fuente más frecuente de la innovación, en algunos casos (innovaciones radicales) puede ser la más importante. En dichos casos, además, la innovación puede partir de investigaciones que se realizan lejos del mercado o, parafraseando a Bush, ajenas a la presión adversa de necesidades comerciales (Bush, 1945). De hecho, existe poca evidencia en la literatura que demuestre que este tipo de investigación no ejerce ningún efecto sobre la innovación, más aún si se tiene en cuenta que en algunos campos (nanociencia, nanotecnología), la ciencia y la tecnología han llegado a ser virtualmente indiferentes una de la otra debido a que sus motivaciones y resultados son cada vez más coincidentes y los que participan en su desarrollo son cada vez más uno y el mismo. En este sentido, tal como lo señala Stokes (1997), el deseo por alcanzar un mejor entendimiento de los fenómenos naturales y las consideraciones relacionadas con la utilidad práctica del conocimiento no constituyen puntos de vista enfrentados. Por el contrario, son dos dimensiones del quehacer científico, las cuales pueden ser desarrolladas simultáneamente sin que una vaya en detrimento de la otra.

Además de lo anteriormente señalado, convendría también tener en cuenta que aún en los casos en los que la innovación parte del conocimiento ya disponible, el desarrollo de actividades científicas puede tener también un efecto importante. En este sentido, por ejemplo, se ha señalado que el desarrollo de actividades de $I+D$, no sólo puede tener un efecto directo sobre la innovación, sino que también contribuye a incrementar la capacidad de la empresa para identificar, asimilar y explotar el conocimiento existente en el entorno. Es lo que se ha denominado en la literatura el papel dual de la I+D, basado en el incremento de la capacidad de absorción (Cohen y Levinthal, 1989). Este argumento puede ser extrapolado a un nivel más amplio y utilizado para analizar la capacidad innovadora de un territorio. De esta forma, los esfuerzos que en materia científica realiza un país pueden servir no sólo para generar nuevos conocimientos que sirvan de base para innovaciones posteriores, sino también para incrementar la capacidad de dicho territorio para hacer un uso más apropiado del conocimiento generado en otros contextos geográficos.

En suma, las características diferenciales de las disciplinas científicas, de los sectores productivos, y el propio ordenamiento socio-institucional de los territorios hacen que la relación ciencia-innovación adquiera diferentes matices. Tal como se ha señalado, dado que no es posible identificar una única forma de hacer ciencia, ni un solo tipo de innovación, tampoco podría hablarse de una única relación ciencia-innovación. Estamos, pues, ante una relación que se caracteriza por su naturaleza compleja y evolutiva.

Lo anteriormente señalado tiene importantes implicaciones, considerando que la visión en torno a la relación ciencia-innovación juega un papel decisivo en la definición de políticas públicas para el desarrollo. Una visión basada en los principios mertonianos de organización de la ciencia y en el modelo lineal de la innovación, conlleva a la definición de políticas basadas en el fortalecimiento de las capacidades científicas y en la implementación de mecanismos que promuevan el desarrollo de actividades de I+D. Éste, de hecho, fue el esquema dominante durante el período posterior a la Segunda Guerra Mundial en la mayor parte de las economías desarrolladas, el cual, además, se guió por el principio general de respeto a la autonomía científica. En contraste, una visión de la relación cienciainnovación basada en los nuevos enfoques sobre producción de conocimiento e innovación, conlleva a la definición de políticas orientadas más hacia el fomento de las relaciones entre productores y usuarios del conocimiento, que a la financiación de la investigación realizada en entornos aislados. Además, estas políticas incorporan instrumentos que inciden sobre el tejido social y económico del territorio (formación de personal, estructura, organización, utiliza- 
ción de nuevas tecnologías), para aumentar su potencial de absorción y utilización de nuevo conocimiento.

La relación ciencia-innovación es un fenómeno que obedece no sólo a la dinámica de las instituciones científicas sino también a las características socio-económicas de su entorno. Aunque parezca evidente, este aspecto ha sido a menudo olvidado en el diseño de las recientes políticas de ciencia e innovación de territorios periféricos, las cuales, guiadas por las experiencias exitosas de otros contextos, han focalizado su atención en el apoyo de transformacio- nes académicas, incidiendo poco en el tejido social y productivo. En este sentido, los gobiernos, que tienen un papel importante como agente facilitador y promotor de estas relaciones, deberian definir estrategias acordes con las condiciones socio-económicas locales. A la luz del análisis realizado precedentemente, más que adoptar uno u otro esquema de intervención, los responsables de formular las políticas de ciencia e innovación deberian desarrollar planteamientos flexibles que se adecuen a las dinámicas científicas y socio-económicas de cada contexto, así como a los problemas que se pretenden resolver.

\section{NOTAS}

1 Aunque comúnmente se interpreta que PLACE constituye un ethos antitético al mertoniano, algunos autores han señalado que contraponerlo al CUDOS puede ser un ejercicio forzado, dado que no tiene un carácter normativo sino más bien descriptivo (Jiménez-Buedo y Ramos, 2009).

2 Para Schumpeter una innovación es posible sin una invención y viceversa, una invención necesariamente no induce a una innovación.

3 Si bien muchas de las taxonomías que se desarrollaron con relación a las fases del proceso innovador consideraban más de tres etapas y empleaban diferentes denominaciones para las mismas, al final la idea central que transmitían podía reducirse a la secuencia general de invencióninnovación-difusión.

4 En respuesta a dicho estudio, la $\mathrm{Na}-$ tional Science Fundation (NSF) Ilevó a cabo el proyecto TRACES (IIT Reserach Institute 1968; Batelle columbus Labs 1973) en el que se analizaron cinco grandes innovaciones. Los resultados de este proyecto fueron completamente opuestos al anterior: la investigación básica era la responsable de más del $70 \%$ de los "eventos" clave para el desarrollo de las innovaciones analizadas. Sin lugar a dudas el perjuicio de los investigadores, los métodos de medición y la interpretación de los resultados, jugaron un papel importante en lo contrastante de los resultados de estos dos proyectos.

5 El pensamiento anterior ha sido reforzado por numerosas investigaciones realizadas por economistas, académicos del ámbito de dirección de empresas e historiadores de la tecnología (Rosenberg, 1994; Henderson y Clark, 1990), en las cuales se ha verificado la gran contribución de las innovaciones incrementales al avance tecnológico.

6 Se reconoce de esta forma un mayor espectro de actividades innovadoras, que no tienen porque circunscribirse al ámbito de la I+D y que no tienen que generarse en la empresa. Por ejemplo, pueden ser sugeridas por los usuarios y los clientes (Rosenberg, 1982; Von Hippel, 1988).

7 El enfoque de sistema de innovación ha llegado a convertirse en uno de los más importantes dentro de la teoría de la innovación y uno de los que más proyección de desarrollo a futuro tiene (Edquist, 2005). Este enfoque, que surgió inicialmen-
Aceptado: 26 de octubre de 2010 
te como un marco de análisis para estudiar y comparar el desempeño de diferentes economías nacionales y determinar la influencia que ejerce en el mismo las estructuras institucionales y productivas (Freeman, 1987; Lundvall, 1992; Nelson, 1993), ha extendido su ámbito de aplicación a otros niveles de análisis, emergiendo de esta forma conceptos como el de los sistemas regionales de innovación (Cooke, 1992; Braczyk et al., 1998), los sistemas sectoriales de innovación (Breschi y Malerba, 1997; Malerba, 2002) y los sistemas tecnológicos de innovación (Carlsson y Stankiewicz, 1995).

\section{BIBLIOGRAFÍA}

Albornoz, M. (2003): "El problema de repensar contextos", Revista CTS, 1 (1), 225-230.

Arocena, R. y Sutz, J. (2001): "La universidad latinoamericana del futuro. Tendencias-Escenarios-Alternativas", Universidad de la República Oriental del Uruguay, Colección UDUAL 11.

Battelle Columbus Labs (1973): Interactions of science and technology in the innovative process: Some case studies, Washington: National Science Foundation.

Braczyk, H.; Cooke, P. y Heidenreich, M. (eds.) (1998): Regional Innovation Systems, UCL Press: London.

Breschi, S. y Malerba, F. (1997): "Sectoral Innovation Systems: Technological Regimes, Schumpeterian Dynamics, and Spatial Boundaries", en Edquist, C. (ed.), Systems of Innovation: Technologies, Institutions, and Organisations, Pinter: London, Washington.

Bush, V. (1945): "Science, the Endless Frontier. A Report to the President", Traducción en Revista de estudios sociales de la ciencia (En REDES 14) (1999): Ciencia, la frontera sin fin, Buenos Aires, 89-136.

Carlsson, B. y Stankiewicz, R. (1995): "On the Nature, Function, and Composition of Technological Systems", en Carlsson, B. (ed.), Technological Systems and Economic Performance: The Case of Factory Specialisation, Kluwer: The Netherlands.

Cohen, W. y Levinthal, D. (1989): "Innovation and learning: the two faces of R\&D", The Economic Journal, 99, 569-596.

Cooke, P. (1992): "Regional Innovation Systems: Competitive Regulation in the New Europe", Geoforum, 23, 365-382.

Edquist, C. (2005): "Systems of Innovation: Perspectives and Challenges", en Fagerberg, J.; Mowery, D. C. y Nelson, R. (eds.), The Oxford Handbook of Innovation, Oxford University Press: Oxford.

Etzkowitz, H. y Leydesdorff, L. (2000): "The Dynamics of Innovation: from National Systems and 'Mode 2' to a Triple Helix of University-Industry-Government Relations", Research Policy, 29 (2), 109-123.

Fernández, M. y Torres, C. (2009): "La ciencia como institución social: clásicos y modernos institucionalismos en la sociología de la ciencia", Arbor, 738, 663-687.

Freeman, C. (1987): Technology Policy and Economic Performance: Lessons from Japan, Pinter Publishers: London and New York.

Freeman, C. (1998): "The economics of technical change", en Archibugi, D. y Michie, J. (eds.), Trade, Growth and Technical Change, Cambridge University Press, [v.c. (2000), La economía de la innovación: las visiones de Ralph Landau y Christopher Freeman. Fundación COTEC, Estudio No 17].
Gibbons, M.; Limoges, C.; Nowotny, H.; Schwartzman, S.; Scott, P. y Trow, M. (1994): The New Production of Knowledge: The Dynamics of Science and Research in Contemporary Societies, London, Sage.

Godin, B. (1998): "Writing Performative History: The New New Atlantis?", Social Studies of Science, 28, 465-483.

Godin, B. (2006): "The linear model of innovation. The historical construction of an analytical framework", Science, Technology \& Human Values, 31 (6), 639-667.

Guston and Keniston (eds.) (1994): The Fragile Contract: University Science and the Federal Government, MIT Press: Cambridge.

Henderson, R. y Clark, K. (1990): "Arquitectural innovation: the reconfiguration of existing product technologies and the failure of established firms", Administrative Science Quarterly, 35, 9-30.

Hessels, L. y Van Lente, H. (2008): "Rethinking New Knowledge Production: A literature Review and a Research Agenda", Research Policy, 37, 740760.

lit Research Institute (1968): Technology in retrospect and critical events in science (TRACES), Washington: National Science Foundation.

Jiménez-Buedo, M. y Ramos, I. (2009): "Más allá de la ciencia académica?: modo 2, ciencia posacadémica y ciencia posnormal", Arbor, 738, 721-737.

Kline, S. J. y Rosenberg, N. (1986): "An Overview of innovation", en Landau, R. y Rosenberg, N. (eds.), The positive sum strategy: Harnessing technology for economic growth, National Academy Press: Washington, 275-306.

Lundvall, B. A. (1992): "User-producer relationships, national systems of innovation and internationalisation", en Lundvall, B. A. (ed.), National Systems 
of Innovation: Towards a Theory of Innovation and Interactive Learning, Pinter: London, 45-67.

MacLaurin, W. R. (1953): "The sequence from invention to innovation and its relation to economic growth", Quarterly Journal of Economics, 67 (1), 97-111.

Malerba, F. (2002): "Sectoral Systems of Innovation and Production", Research Policy, 31, 247-264.

Mansfield, E. (1968): The economics of technological change, New York: W. E. Norton.

Martin, B. R. (2003): "The changing social contract for science and the evolution of the university", en Geuna, et al. (ed.): Science and Innovation: Rethinking the rationales for funding and governance, 7-29, Cheltenhan: UK.

Martin, B. y Etzkowitz, H. (2000): "The origin and evolution of the university species", SPRU, Electronic Working Paper Series, 59.

Merton, R. (1942): "Science and technology in a democratic order", Journal of Legal and Political Sociology, 1, 115126. (Reimpreso como: "The normative structure of science", en R. K. Merton, The Sociology of Science, University of Chicago Press, Chicago, 1973).
Mitroff, I. (1974): "The Apollo Moon Scientist: A case study of the ambivalence of scientists", American Sociological Review, 39, 579-595.

Mulkay, M. (1976): "Norms and ideology in science", Social Science Information, 15, 637-656.

Myers, S. y Marquis, D. (1969): "Successful industrial innovations: A study of factors underlying innovation in selected firms", NSF 69-17. Washington, D.C: National Science Foundation.

National Science and Technology Council (1999): "Nanotechnology Research Directions: Vision for Nanotechnology in the Next Decade", Executive Office of the President, Washington D.C.

Nelson, R. (ed.) (1993): National Innovation Systems: A Comparative Analysis, Oxford University Press: New York, Oxford.

Nowotny, H.; Scott, P. y Gibbons, M. (2001): Re-Thinking Science. Knowledge and the Public in an Age of Uncertainty, Cambridge, Reino Unido: Polity Press.

Rosenberg, N. (1982): "How exogenous is science?", en Rosenberg, N. (ed.), Inside the black box: Technology and economics, Cambridge University Press, Cambridge, 141-159.
Rosenberg, N. (1994): "Incertidumbre y cambio tecnológico", Revista de Historia Industrial, 6, 11-30.

Schumpeter, J. A. (1939): Business Cycles: A Theoretical, Historical and Statistical analysis of the Capitalism Process, New York, McGraw-Hill.

Sklair, L. (1972): "The political sociology of science", The Sociological Review Monograph, 18, 43-59.

Stokes, D. (1997): Pasteur's Quadrant: Basic Science and Technological Innovation, Brookings Press, Washington D.C.

U.S. Department of Defense (1969): Project hindsight final report, Washington, D.C: Office of the Director of Defense Research and Engineering.

Utterback, J. (1974): "Innovation in industry and the diffusion of technology", Science, 183, 620-626.

Von Hippel, E. (1988): The Sources of Innovation, Oxford University Press: Oxford.

Weingart, P. (1997): "From 'Finalization' to 'Mode 2': Old Wine in New Bottles?", Social Science Information, 36, 591613.

Ziman, John (2000): Real Science: What It is and What It Means, Cambridge, UK: Cambridge University Press. 